\title{
Molecular dynamics simulation for self-diffusion coefficients of ginger bioactive compounds in subcritical water with and without ethanol
}

\begin{abstract}
Molecular dynamics simulation was used to calculate the self-diffusion coefficients of ginger bioactive compounds (6-gingerol and 6-shogaol) in subcritical water with the presence of ethanol as an entrainer (0-10 mol\%) at temperatures from 373.15 to $453.15 \mathrm{~K}$. The all-atom optimised-potentials (OPLS/AA) were employed for the ginger bioactive compounds and ethanol. The extended simple point charge (SPC/E) model was adopted for water molecules. The self-diffusion coefficients increase from $1.00 \times 10^{-9}$ to $2.70 \times 10^{-9} \mathrm{~m}^{2} / \mathrm{s}$ with increasing temperature from 353.15 to $453.15 \mathrm{~K}$. The self-diffusion coefficients also increase from $1.71 \times 10^{-9}$ to $3.00 \times 10^{-9} \mathrm{~m}^{2} / \mathrm{s}$ with increasing percentage of ethanol from 0 to $10 \mathrm{~mol} \%$ at $413.15 \mathrm{~K}$. The radial distribution functions between the ginger bioactive compounds and subcritical water (solvent) illustrate a weak interaction between the ginger bioactive compounds and solvent. The interaction increases with addition of ethanol as entrainer.
\end{abstract}

Keyword: Extraction process; Subcritical water; Self-diffusion coefficient; Ginger bioactive compound; Entrainer; Ethanol 